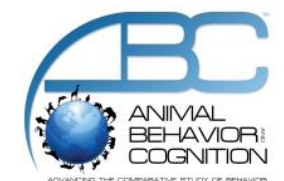

\title{
Differential GO and NOGO Learning Within an Auditory Discrimination Task
}

\author{
Kusuma Anand ${ }^{1,2}$ and Paul M. Nealen ${ }^{1 *}$ \\ ${ }^{1}$ Department of Biology, Indiana University of Pennsylvania, Indiana, PA \\ ${ }^{2}$ Department of Neurobiology and Behavior, Cornell University, Ithaca, NY \\ *Corresponding author (Email: pnealen@iup.edu)
}

Citation - Anand, K., \& Nealen, P. M. (2019). Differential GO and NOGO learning within an auditory discrimination task. Animal Behavior and Cognition, 6(2), 141-157. https://doi.org/10.26451/abc.06.02.05.2019

\begin{abstract}
Animal discrimination training and testing often are used to assess animal behavioral flexibility and capabilities. GO-NOGO discrimination, in particular, has been used extensively, across a multitude of species and experimental paradigms. In its typical form, GO-NOGO discrimination learning is assessed by the magnitude of, and rate of acquisition of, discrimination capability, which is nominally low at the start of training, and through experience, is improved as a function of the reward schedule employed. Discrimination performance in this task is normally reported via a single empirical metric, as the percentage of stimulus-response trials which included a 'correct' response. However, this view of discrimination as a singular skill masks the considerable differences which exist in GO and NOGO responses (which typically require an action, and a suppression of action, respectively). To investigate the potential for these two responses to be learned independently and via different mechanisms, we performed operant training of adult zebra finches (Taeniopygia guttata) in a GO-NOGO auditory discrimination task. We demonstrate that correct responsiveness to GO and NOGO stimuli was learned via different overall performance trajectories, with performance on GO and NOGO trials emerging independently both within and between training sessions. We propose that this differential learning derives from a combination of response generalization and response modal form. These data suggest that response action and suppression are subserved by different neural circuits, and further indicate that consideration of discrimination tasks as univariate problems masks underlying complexity by obscuring their individual components.
\end{abstract}

Keywords - Auditory discrimination, GO-NOGO, Zebra finch, Operant, Learning

Animals are subject to a broad array of sensory signals, many of which they intercept and subject to neural processing and psychological evaluation. Instinct shapes much of animal response to critical environmental stimuli (such as cues of nearby predators, or dangerous physical conditions), but for stimuli of less-immediate survival consequences, experience and learning also contribute to responsiveness, often with regards to the consequences any particular response may engender (Skinner, 1963). This 'trial-and-error' learning has long been adopted for laboratory study using operant conditioning techniques and has shed much light on the capabilities by which animals obtain learned responsiveness to stimuli, and the constraints that exist on their doing so.

While discrimination training may be implemented in a variety of paradigms, 'GO-NOGO' methodology is perhaps the most common form of learned discrimination subject to routine operant study. In its typical form, GO-NOGO discrimination requires a subject to learn differential responses to two different sensory stimuli, with one response ('GO') representing an active behavior (such as a movement, or key press) while the other response ('NOGO') requires a suppression of that same 
behavior. As is the case for many implementations of discrimination training, descriptions of GO-NOGO discrimination capability often rely upon a single or a relatively few common measures of learned responsiveness (such as learning speed, or percent accuracy; examples in Cynx, 1993; Kraemer \& Apfelbach, 2004; Remington, Osmanski, \& Wang, 2012; Scharff, Nottebohm, \& Cynx, 1998; Schellinck, Brown, \& Slotnick, 1991), and typically consider collective outcomes measures that group GO and NOGO trial responses. This is despite the fact that multiple types of neurophysiological assessments have revealed GO and NOGO task performances to be neurally distinct at several scales, including individual neuron activity patterns (Blake, Strata, Churchland, \& Merzenich, 2002; de Haan et al., 2018; Kalaska \& Crammond, 1995), neural circuit-level activation (Ahmadi et al., 2013; Criaud \& Boulinguez, 2013; Schiff et al., 2018) as well as in event-related-potential (ERP) neuronal population signatures (Nieuwenhuis, Yueng, van den Wildenberg, \& Ridderrinkhof, 2003; Simson, Vaughan, \& Ritter, 1977). As such, a singular focus upon improved overall discrimination performance is likely to obscure the components of learned performance improvements.

That discrimination performance is often viewed, and reported, as a singular capability masks the potential for this learned responsiveness to be comprised of multiple elements (as demonstrated in Gess, Schneider, Vyas, \& Woolley, 2011). If correct responsiveness to GO and NOGO stimuli requires different behaviors, it may also involve different neural systems for response learning and performance. While mechanistic evaluation of neuronal activity provides the most direct route to an understanding of the differential learning that occurs to facilitate the acquisition of discrimination capability, phenomenological evaluations of the development of discrimination performances can reveal subtleties that bear further investigation. We report one such evaluation here, of the differential progress achieved within avian auditory GO and NOGO learned responsiveness.

Many laboratory studies of operant learning have used passerine birds, favored for their small size, ease of care, and complex behavioral capabilities. Because passerines are vocal learners, much investigation has focused upon the role of learned song in communication, and many investigations of avian discriminatory capabilities related to song have been conducted. Song discrimination studies of a variety of passerine species have revealed a high degree of discrimination capability, rapid rates of learned responsiveness, and subtle differences in discrimination learning relative to gender, season, and familiarity (Cynx, 1993; Cynx \& Nottebohm, 1992; Cynx, Williams, \& Nottebohm, 1992; Kriengwatana, Spierings, \& ten Cate, 2016; Okanoya \& Dooling, 1991a,b; Riebel \& Slater, 1998; Verzijden, Etman, van Heijningen, van der Linden, \& ten Cate, 2007). These studies demonstrate that auditory discrimination in passerines is highly refined, essential for their social navigation, and behaviorally plastic. As such, it represents a very useful experimental model for investigation of behavioral learning.

Recently, Gess et al. (2011) reported the development of an automated operant training platform for auditory GO-NOGO discrimination and its successful use in zebra finches. Their study highlights the behavioral complexity and capability associated with passerine auditory communication, as individuals rapidly acquired a primary discrimination task, and showed high levels of learned discrimination transference to other sets of stimuli. Gess et al. reported individual differences in learned acquisition of discrimination and suggested that these differences may be due to individual biases in response to test stimuli. Gess et al. also demonstrated that the GO and NOGO components of an auditory discrimination task may be achieved via different learning trajectories, and they presented select examples of GO and NOGO trial performance progressing independently and at different rates, a finding which prompted the current study.

Here, we have conducted similar operant training for auditory discrimination in zebra finches, with the goal of clarifying the form, timing, and independence of learning of the individual GO and NOGO components of the task, as well as their within and between-training session maintenance. We focus upon response error reduction during training (e.g., the reduced percentage of "incorrect" responses), and quantify the magnitude of both within-session ("daily") changes in task performance as well as between-session ("overnight") regressions in performance, for their ability to contribute to differential evolution of correct GO and NOGO responsiveness. Our results suggest that differential GONOGO discrimination learning is driven by a combination of two primary factors: subject model 
responsiveness to stimuli (as a function of task familiarity and subject willingness to engage) and stimulus generalizability (as a function of the degree to which stimuli are successfully discriminated). The combination of these two factors produces gradual improvements in overall discrimination performance, but as a function of independent improvement in learned GO and NOGO responsiveness, which themselves are subject to differing degrees of daily and overnight neural maintenance.

\section{Methods}

\section{Ethics Statement}

All animal housing, care, and use was performed with the permission of the Indiana University of Pennsylvania Institutional Animal Care and Use Committee (IACUC log \#02-1415).

\section{Subjects}

Adult zebra finches (Taeniopygia guttatta), were obtained from an in-house colony. Study subjects originally were housed in the colony either as mated pairs or in small, single-sex groups. While in colony housing, white millet seed and water were available ad libitum. Weekly food supplements included hard-boiled egg and lettuce. The avian colony was maintained on a 14L:10D light cycle, yearround. Twenty-two subjects $(8 \mathrm{~F}, 14 \mathrm{M})$ were individually trained in either of two identical, customassembled operant conditioning chambers modeled after those used successfully by Gess et al. (2011). Two male subjects were withdrawn from analyses after failing to successfully complete training.

\section{Apparatus}

Each operant training/testing chamber consisted of a $12 \times 11 \times 9.5$ in wire mesh and acrylic panel cage, containing a water bottle (accessible at any time), a perch, an actuated feeder, and an infra-red (IR) response trigger (Figure 1). The operant chambers each were held within larger acoustic attenuation chambers that provided complete visual and partial auditory isolation from other subjects. Within the larger chamber, a fluorescent light, a monaural speaker, and a CCD camera were arrayed. All components were modeled after Gess et al. (2011). The feeder, trigger, light, and speaker were controlled by custom (Gess et al., 2011) MATLAB code (MathWorks, Inc.; Natick, MA), generously provided by Sarah Woolley and David Schneider (Columbia University, New York, NY). The camera was controlled by Geovision software (Geovision, Inc.; Taipei, Taiwan) to observe and record training/testing sessions.

\section{Design and Procedure}

Training sessions lasted five hours each day, with most birds trained five days per week. Training sessions were initiated at morning 'lights-on' such that subjects were naturally fasted during the overnight period before training. At the end of weekday training, chamber doors were opened to allow subjects auditory and visual access to the avian colony. Birds remained in-chamber overnight between successive training sessions, and normally were returned to colony housing over weekends.

Training occurred in three distinct phases, modeled after Gess et al. (2011). The first phase of training (Acclimation) was designed to allow the subjects to gain familiarity with the training apparatus. On the first day of Acclimation, subjects had ad libitum access to food and water. Thereafter, food access was software-controlled, via the cycling of the seed feeder into the chamber for a fixed duration, followed by an interval of food unavailability (randomized between 30 and $60 \mathrm{~s}$ ) during which the seed feeder cycled out of the chamber. The duration of feeder availability was shortened from intervals of $600 \mathrm{~s}$ to intervals of $7 \mathrm{~s}$ (Table 1), over successive days of Acclimation training. The 8 days of Acclimation training allowed subjects to become comfortable with the chamber and the movements of the seed feeder into/out of the chamber. 
Table 1

Timing Parameters for the Phases of Operant Training.

\begin{tabular}{|c|c|c|c|c|c|c|}
\hline Training phase & Condition & Stage & $\begin{array}{l}\text { Feeder availability } \\
\text { duration (seconds) }\end{array}$ & \multicolumn{2}{|c|}{ Inter-trial interval (seconds) } & $\begin{array}{c}\text { Duration lights } \\
\text { out (seconds) }\end{array}$ \\
\hline & Stationary Feeder & ----- & Always Available & ------ & ------- & ------ \\
\hline Acclimation & Moving Feeder & 1 & 600 & 30 & 60 & ------- \\
\hline Acclimation & Moving Feeder & 2 & 300 & 30 & 60 & ------- \\
\hline Acclimation & Moving Feeder & 3 & 150 & 30 & 60 & ------- \\
\hline Acclimation & Moving Feeder & 4 & 75 & 30 & 60 & ------- \\
\hline Acclimation & Moving Feeder & 5 & 30 & 30 & 60 & ------- \\
\hline Acclimation & Moving Feeder & 6 & 15 & 30 & 60 & ------- \\
\hline Acclimation & Moving Feeder & 7 & 7 & 30 & 60 & ------- \\
\hline Shaping & Visual Target & 1 & 10 & ----- & ------ & -------- \\
\hline Shaping & No Target & 2 & 10 & ------- & ------- & ------- \\
\hline $\begin{array}{l}\text { Discrimination } \\
\text { Training }\end{array}$ & ------ & ------- & 6 & ------- & ------ & 16 \\
\hline
\end{tabular}

Once subjects were successfully eating from the actuated feeder, they were moved to the Shaping phase of training, which required subjects to activate the IR trigger to make the feeder available for a brief $(10 \mathrm{~s})$ duration. Subjects triggered food availability by pecking at the visual target behind the yoke of the infra-red (IR) trigger switch (Figure 1). No limit was placed upon the number or rate of feeder triggers during this Shaping phase of training, which allowed subjects to learn to use the IR trigger switch to make the seed feeder available. Subjects were deemed to have successfully completed the Shaping phase of training when review of Shaping session video files revealed that subjects had successfully triggered, and then eaten from, the actuated seed feeder a minimum of 20 times during a $5 \mathrm{hr}$ training session. Following Shaping, subjects were advanced to the Discrimination phase of training.

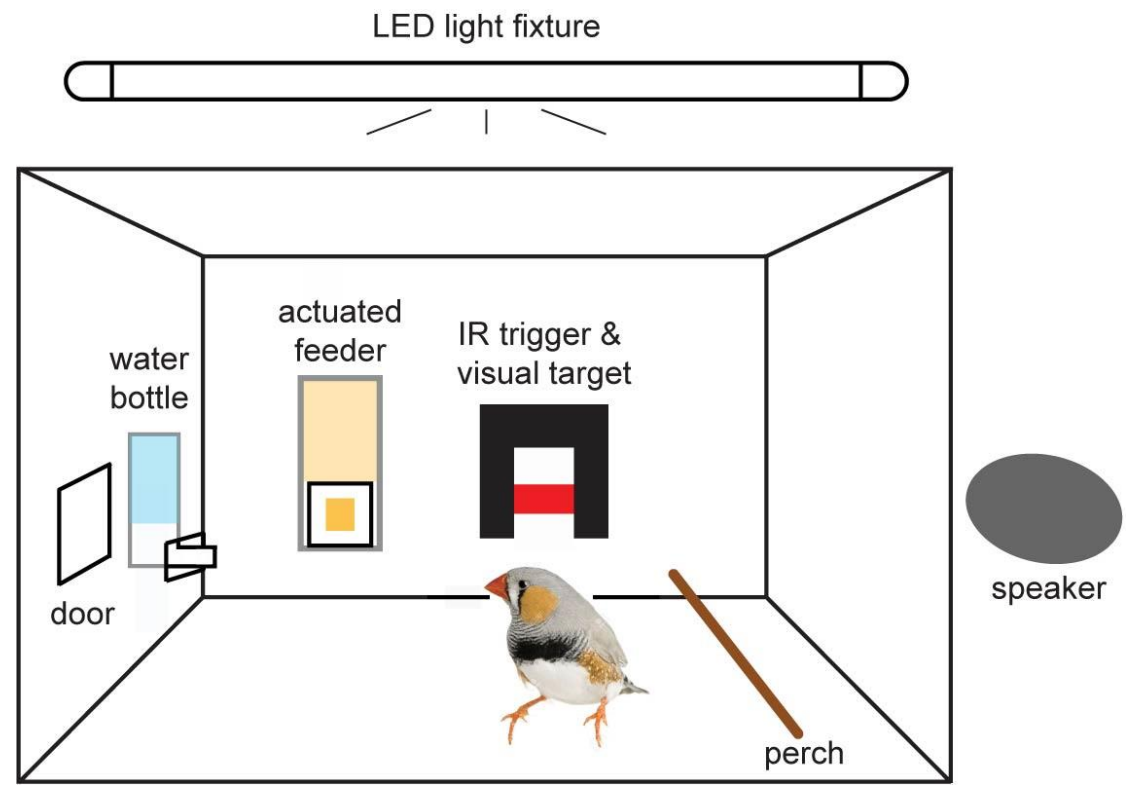

Figure 1. Generalized schematic of the operant chambers used. Each chamber was outfitted with a perch and water bottle (always available), as well as an actuated seed feeder which was controlled via the operant training and testing software. Subject responses were detected by an infra-red (IR) trigger, operated by subject pecking at or near to a colored target located against the cage wall. Auditory stimuli were made available via a calibrated speaker adjacent to the chamber, while overhead lighting was provided by a fluorescent-style LED light bar. 
Relative amplitude
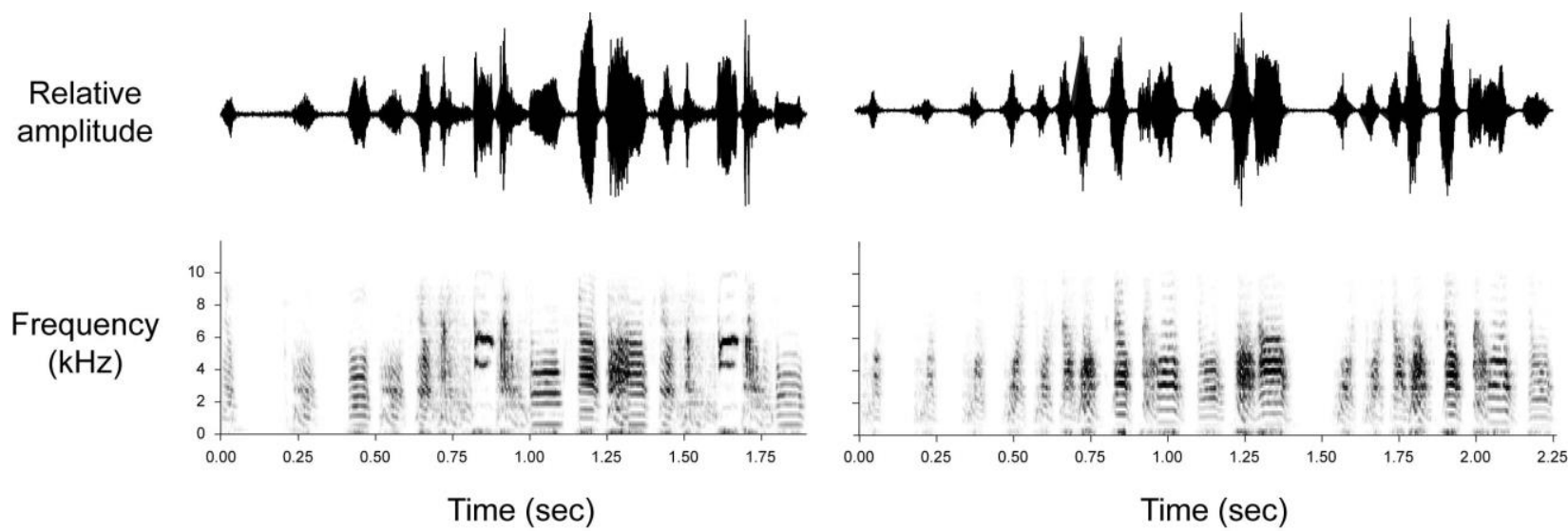

Figure 2. The two sound stimuli used during auditory discrimination training (left and right figure columns). Each stimulus is the learned song of an adult male zebra finch unfamiliar to the training subjects. Each sound stimulus consists of two complete song motifs, preceded by introductory notes (Sossinka \& Boehner, 1980). Oscillograms (top row) depict relative amplitude while sonograms (bottom row) depict the time-frequency relationship for each stimulus.

During the Discrimination training phase, subjects learned to discriminate between two initiallyunfamiliar sound stimuli, each comprised of a single adult male zebra finch song (Figure 2). Each song contained several motifs (sets of repeated syllables; Sossinka \& Boehner, 1980) from the learned song of a different (conspecific) male zebra finch; both song stimuli were entirely unfamiliar to the testing subjects. All subjects were trained on the same pair of songs, chosen for their relatively high degree of acoustic dissimilarity. Training was of typical GO-NOGO form, in that learned responsiveness to the 'GO' stimulus required the subject to activate the IR trigger when the GO stimulus was heard, while learned responsiveness to the NOGO stimulus required the subject to withhold activation of the IR trigger upon audition of the NOGO stimulus. Although all subjects were trained using the same two sound stimuli, individual sound stimulus assignment as the 'GO' or 'NOGO' stimulus was alternated among training subjects.

Two response options for each of the two stimulus categories resulted in four possible outcomes for a GO-NOGO discrimination trial (Table 2), with subjects learning over time to provide the appropriate response for a given stimulus as a function of response contingencies. The majority $(n=17)$ of birds were trained via 'normal' outcome contingencies, such that correct responses to the GO stimulus ('Hit' trials) were rewarded with food access while incorrect responses to the NOGO stimulus ('False alarm' trials) resulted in a brief period of lights-out (Table 1). Failure to respond to the GO stimulus and a lack of response to the NOGO stimulus resulted in 'null' consequences, with neither food access nor lights-out being delivered. To confirm the ability of outcome contingencies to shape learning, three birds were trained with 'reverse' contingencies (Table 2) which resulted in a rapid cessation of responses to training stimuli (see Results).

Table 2

Operant Training Response Matrices and the Reward Schedules Employed

\begin{tabular}{|c|c|c|c|c|c|c|c|c|}
\hline \multirow[t]{2}{*}{ Stimulus } & \multirow[t]{2}{*}{ Response } & \multirow[t]{2}{*}{ Designation } & \multicolumn{3}{|c|}{ Normal Contingencies } & \multicolumn{3}{|c|}{ Reverse Contingencies } \\
\hline & & & Reward & Punishment & Null & Reward & Punishment & Null \\
\hline GO Stimulus & Activate Sensor & Hit & $\mathrm{X}$ & & & & & $\mathrm{O}$ \\
\hline GO stimulus & Withhold Sensor & Miss & & & $\mathrm{X}$ & & $\mathrm{O}$ & \\
\hline NOGO stimulus & Moving Feeder & False Alarm & & $\mathrm{X}$ & & & & $\mathrm{O}$ \\
\hline NOGO stimulus & Moving Feeder & Correct Reject & & & $\mathrm{X}$ & $\mathrm{O}$ & & \\
\hline
\end{tabular}




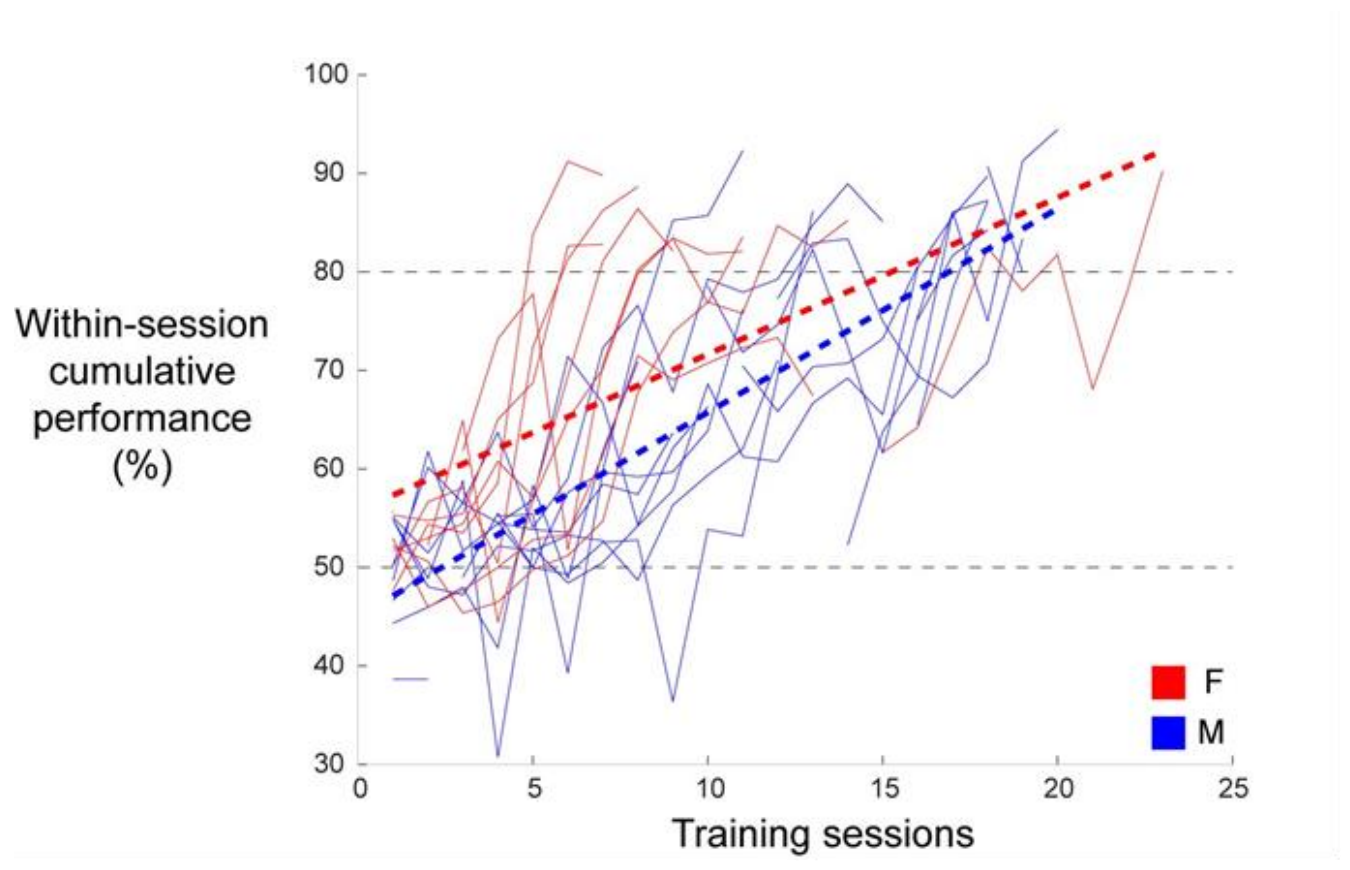

Figure 3. Discrimination acquisition curves for female (red) and male (blue) subjects. Thin lines depict individual subject learning curves across training sessions, plotted as the (within-day) cumulative percentage of discrimination trials (GO and NOGO combined) which included correct responses. Heavy dashed lines represent male and female group linear regressions; thin dashed lines depict nominal error level at the start of training (50\%) as well as the discrimination training criterion of $80 \%$ correct responses.

During discrimination training, subjects initiated discrimination trials via activation of the IR trigger. Thus, subjects controlled their own work rate. Because subjects were fasted overnight before daily training, they typically initiated discrimination trials at a steady rate. Subjects routinely performed > 100 discrimination trials during $5 \mathrm{hr}$ training sessions (subject grand mean: 129 trials/session; range: 37 278 trials/session) and an average of 1,630 trials throughout the course of discrimination training (range: $705-3,892$ discrimination trials per individual). Training sessions that included $<10$ discrimination trials (18/363 sessions, $<5 \%$ of all sessions) were excluded from analysis. Discrimination training ended when a subject's within-session cumulative discrimination performance rate exceeded $80 \%$ correct at the end of three separate daily training sessions (consecutive, or otherwise; see Figure 3).

\section{Data Analyses}

All raw data were tabulated in Microsoft Excel (Microsoft Corp.; Redmond, WA). Data computation and graphical analyses were completed in MATLAB (MathWorks, Inc.; Natick, MA) while statistical assessments were performed in SPSS (IBM Corp.; Armonk, NY). The primary measure of learning was discrimination performance rate, measured as the proportion of trials that included a correct response (GO or NOGO) for a given stimulus. Dependent measures included end-of-day cumulative discrimination performance rate (as an indication of the daily progression of learned discrimination capability), instantaneous performance rate (computed over successive blocks of 5 discrimination trials, to assess how learned discrimination evolved during training sessions), and within- and between-day changes in performance rates (to allow evaluation of the magnitude and direction of within- and betweenday changes in discrimination performance). Because the number of discrimination trials per session and the number of training sessions varied among subjects, within- and across-day training progression (trial number within a day, training day number within overall training) were normalized between 0 and 1 for some analyses, allowing creation of a three-dimensional 'error surface' (a 51-point X 51-point, 3- 
dimensional grid; see Figure 4). Other analyses evaluated across-day changes in discrimination capability by dividing training into three equal periods ('early,' 'middle,' and 'late' training). One-way ANOVA was used to assess gender differences in performance as well as within-session changes in responsiveness. 2-way ANOVA was used to examine the effect of stimulus identity on response outcomes across training periods. Because subject error rates were initially high and because a low error rate was used as discrimination learning criterion, analyses focused upon the gradual reduction in GO-NOGO response error over the course of discrimination training. So that we could isolate potential differences in GO versus NOGO responses, the majority of analyses included these two response classes as a variable of interest. This typically meant evaluating performance on GO trials separately from NOGO trials.

\section{Results}

Subjects completed Shaping training over an average of 11.8 days (range: $4-28$ days); female and male subjects did not differ in their durations of Shaping training $(F(1,20)=2.127, p=.160)$.

\section{Overall Acquisition of Discrimination Capability}

As demonstrated previously by Gess et al. (2011), this training regimen successfully induced auditory discrimination learning and capability in adult zebra finches of both sexes. The 17 subjects $(8 \mathrm{~F}$, $9 \mathrm{M})$ trained under the standard response contingencies successfully achieved discrimination criteria in an average of 14 training sessions (range: 7 to 22 sessions; Figure 3). Females tended to learn the discrimination faster than did male subjects (averages of 11 and 16 training sessions, respectively; $F(1$, 16) $=6.91, p=.019, \eta_{\mathrm{p}}^{2}=0.315$ ). Although males and females acquired discrimination capability at different overall rates, all subjects of both sexes exhibited the same differential trajectories for GO and NOGO learned responsiveness (described below). As differences between GO and NOGO learning were our focus, subjects of both sexes were pooled for assessments of learning patterns (below).

\section{Subject GO and NOGO Responses Emerged Differently During Training}

To examine how training shaped learning, normalized training data were compiled across subjects for examination of discrimination response error surfaces, designed to reveal changes in training error both within and across training sessions. Figure 4 depicts discrimination trial error surface plots, for both an individual male subject (left column) and for the collective group of trained subjects ( $n=17$; right column). As expected, when GO and NOGO trials were analyzed collectively, overall discrimination response error rates (from 0.0 to 1.0 proportion of trials) were highest at the start of subject training, and fell slightly within training sessions, and more moderately across training sessions, until discrimination criterion was achieved (Figure 4, top row). Independent assessment of GO and NOGO trial responses (Figure 4, middle and bottom rows, respectively), however, revealed that progress in performance was achieved very differently for these two types of responses. GO trial response error rates were highest at the start of training and decreased steadily both within (lower right axis in each subplot) and across (lower left axis in each subplot) training days. In contrast, NOGO trial response error rates were low early in training, rose both within and across training days through the middle of training, and then fell to criterion as training concluded. All individuals trained on the standard response contingencies (described in Table 2) exhibited this general pattern of response (Figure 4, right column), even while individual learning trajectories incorporated some temporal instability (individual example in Figure 4, left column).

\section{Changes in Response Error Within Training Days}

Discrimination learning is commonly assessed by evaluation of end-of-day cumulative discrimination performance (as in Figure 3). We have demonstrated differences in across-day patterns of 


\section{Single bird example}
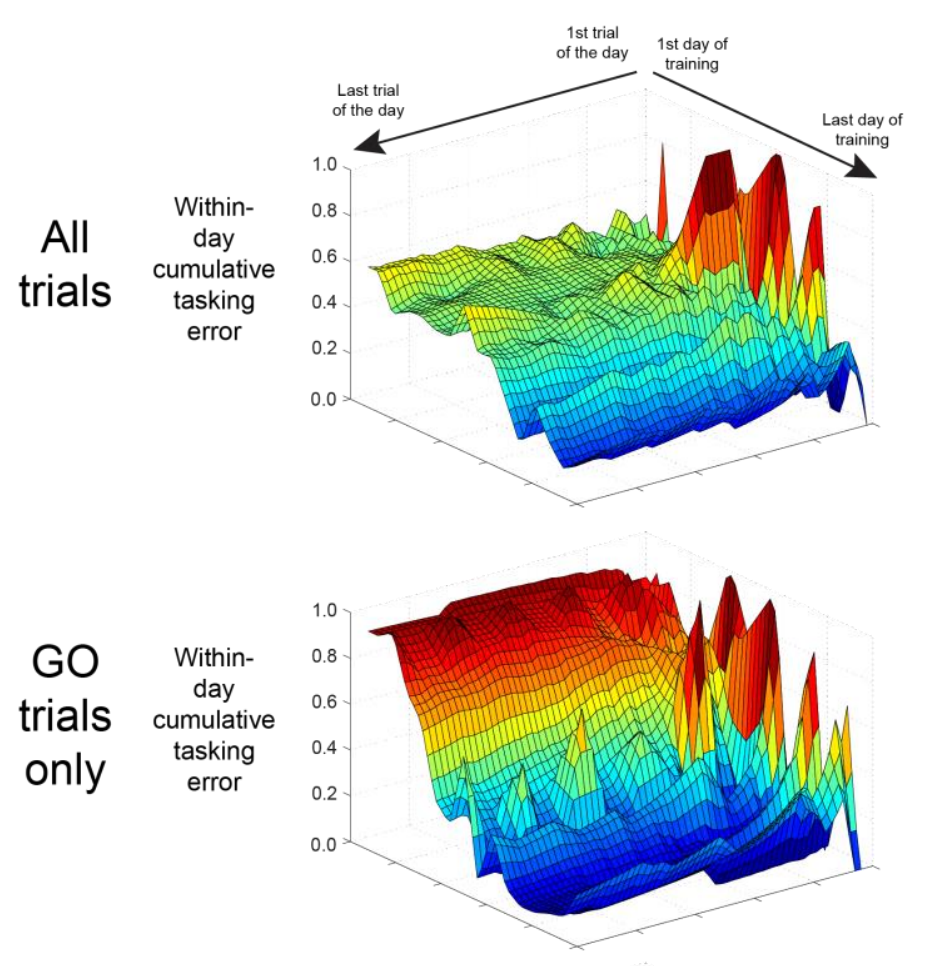

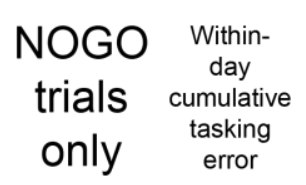

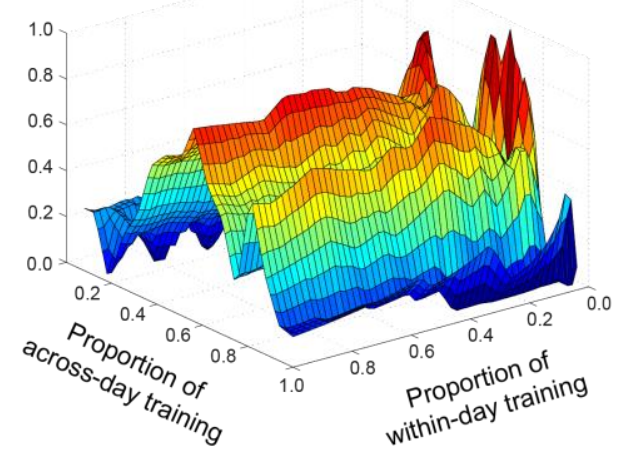

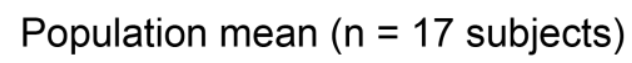
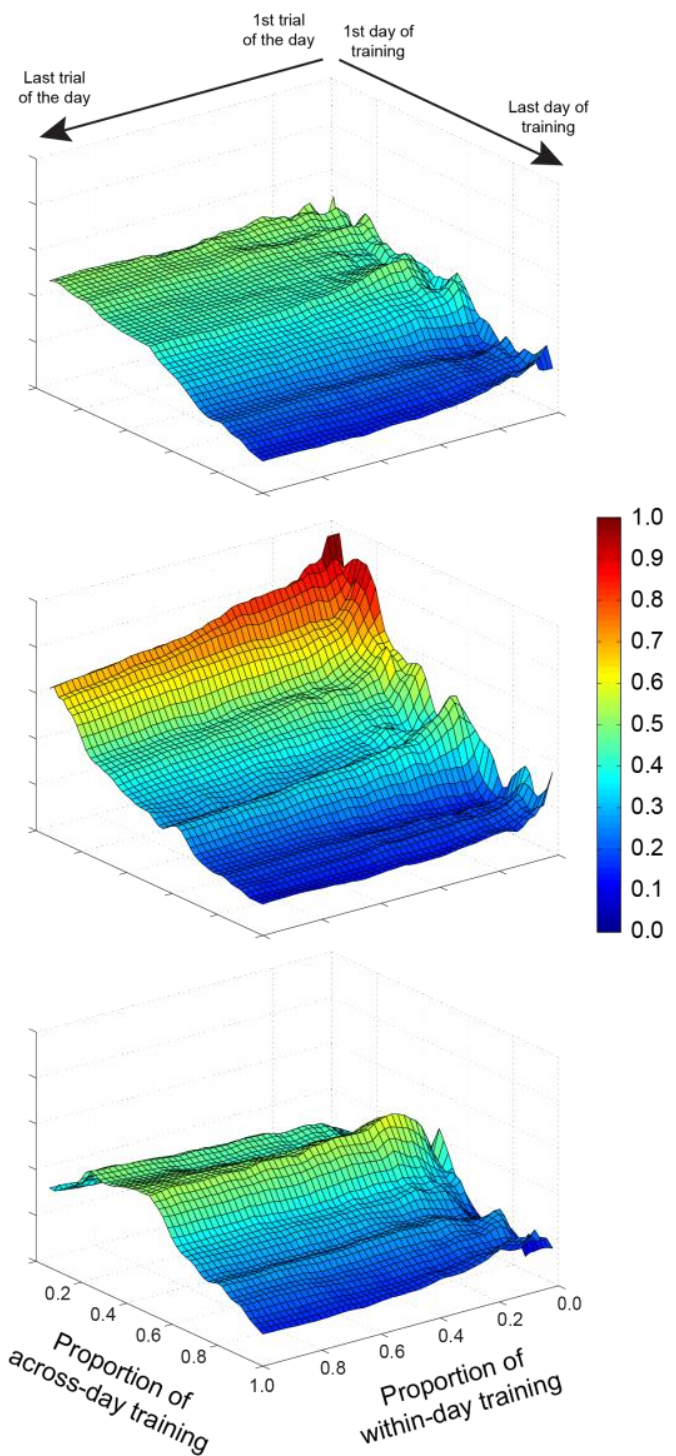

Figure 4. Discrimination error reduction over the course of subject training, for GO and NOGO trials considered together (top row) and for GO (middle row) and NOGO (bottom row) trials evaluated independently. Error response surfaces are presented for both a single bird example (left column) as well as for the collective group of trained subjects $(\mathrm{n}=17$; right column).

GO and NOGO trial response errors (Figure 4), and note that they were driven, in part, by differential patterns of within-day changes in performance. Figure 5 depicts grouped data revealing how cumulative response error changed within daily training sessions, as subjects progressed across days in their training. End-of-daily training session cumulative response error (red lines) closely matched daily start-of-training session response error (green lines) when both GO and NOGO discrimination trials were considered together $\left(F(1,94)=2.031, p=.16, \eta_{\mathrm{p}}^{2}=0.21\right.$; Figure 5, left), but GO and NOGO trial response errors changed significantly during the course of individual training sessions. GO trial response error was highest at the start of daily training sessions, and fell during daily training $(F(1,94)=19.513, p<.001$, $\eta_{\mathrm{p}}{ }^{2}=0.172$; Figure 5, middle), even as it trended downward across training sessions. In contrast, NOGO trial response error was at its lowest at the start of daily training, and rose through the day to peak at end of daily training $\left(F(1,94)=75.521, p<.001, \eta_{\mathrm{p}}{ }^{2}=0.445\right.$; Figure 5 , right $)$, as well as first rising and then 

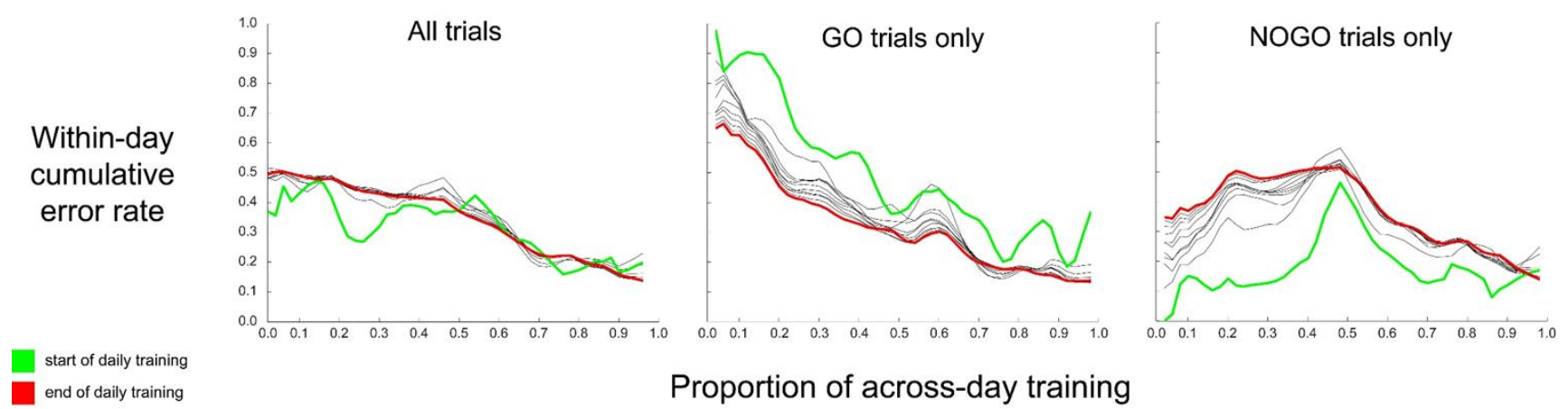

Proportion of across-day training

Figure 5. Differential evolution of response errors within and across training sessions. Depicted here are grouped data (mean of 17 subjects) for discrimination trial response error ( $\mathrm{Y}$ axis, from $0-1$ proportion of trials) at the start of daily training sessions (green lines) and the end of daily training sessions (red lines). Thin black lines depict 30 min intervals throughout daily training.

falling across daily training sessions. Because these differential GO and NOGO within-day patterns opposed each other, collective discrimination error (left panel) evolved little during the course of daily training. These differential patterns of daily response emergence held even as the overall responses evolved toward lower error levels as training progressed across training days (e.g., all error curves trend downward during the final days of training to a minimum at the overall end of training).

\section{Incomplete Overnight Maintenance of Daily Changes}

In theory, steady change in discrimination performance gained during the course of a single training session could lead to rapid, long-term gains in performance, if daily performance improvements were faithfully retained from one training session to the next. To evaluate the degree to which daily performance gains were maintained across training sessions, the instantaneous rate of discrimination performance (assessed over five successive discrimination trials) was compared at the start and end of daily training sessions, as well as from the end of one training session to the start of the next session.

The majority of training sessions $(159 / 215 ; 74 \%)$ were on sequential days, and thus were separated by only one overnight period between sessions. 17\% of sessions (37/215) were separated by 2 to 3 days, while $5 \%$ of sessions $(11 / 215)$ were separated by 4 to 5 days and $4 \%$ were separated by more than 5 days (8/215). The magnitude of between-session change in task performance did not vary as a function of the number of intervening overnight periods (from 1 to 5 overnight spans) for either GO trials (Pearson $r=-.589, n=5, p=.19$ ) or for NOGO trials $(r=.78, n=5, p=.12)$, so training sessions separated by single overnight spans were considered together with those separated by 2 to 5 overnight spans.

In the intervening period between training sessions ("overnight"), both GO and NOGO trial performances changed in a manner that partially countered their within-day changes. Both types of responses were most plastic early in training, with smaller magnitudes of change over the middle and late stages of training. As reported above, GO trial performance improved over the course of daily training sessions (within-day cumulative performance; Figure 5, middle). This effect is observable in assessment of GO trial instantaneous performance rate as well (Figure 6, top). During the first third of daily discrimination training sessions ('early' training), GO trial instantaneous error rates dropped an average of $25 \%$ during daily training sessions, with smaller average daily improvements during the middle (-17\%) and latter $(-3 \%)$ days of discrimination training. The within-day improvement in GO trial performance, however, was largely lost overnight between training sessions (Figure 6, bottom). During the first third of daily discrimination training ('early' training), GO trial instantaneous performance rates worsened overnight by $15 \%$, with, as before, smaller changes apparent during the middle $(13 \%)$ and latter $(1 \%)$ thirds of training. Thus, net daily gains in performance were steadily realized across training sessions (as depicted in Figure 3), but the magnitude of net daily gains in GO trial discrimination performance was only a fraction of total daily gains. 
As described above, NOGO trial performance worsened during the course of daily training (NOGO trial within-day cumulative performance; Figure 5, right). Here too this result was confirmed in assessment of instantaneous performance rates (Figure 6, top). The within-session change in NOGO trial instantaneous error rate was $+30 \%$ during early training days, with smaller average effects seen during mid $(+8 \%)$ and late $(<+1 \%)$ training days (Figure 6). As was seen for GO trial performance, overnight changes in NOGO instantaneous error rates opposed within-day performance changes, with NOGO trial instantaneous error rates improving by average magnitudes of $-26 \%,-12 \%$, and $-5 \%$ during early, mid, and late daily training, respectively (Figure 6, bottom). As seen in the results of training for GO responses, daily changes in NOGO performance were partially offset by overnight shifts in performance, but the direction of daily and overnight changes in GO and NOGO response performance opposed one another. Repeated measures, two-way ANOVA revealed that, collectively, daily changes in instantaneous error rate did not differ by training stage $\left(F(2,32)=1.937, p=.161, \eta_{\mathrm{p}}^{2}=0.108\right)$, but differed dramatically by stimulus category (GO vs. NOGO; $\left.F(1,16)=56.146, p<.001, \eta_{\mathrm{p}}{ }^{2}=0.778\right)$. Because GO and NOGO responses were changing in opposing directions during daily training, the (training stage*stimulus category) interaction term was highly significant $\left(F(2,32)=18.726, p<.001, \eta_{\mathrm{p}}{ }^{2}=\right.$ 0.539). Similarly, overnight changes in instantaneous error rates did not systematically differ by training stage $\left(F(2,32)=1.496, p=.239, \eta_{\mathrm{p}}^{2}=0.086\right)$, but differed dramatically by stimulus type (GO vs. NOGO; $\left.F(1,16)=30.877, p<.001, \eta_{\mathrm{p}}^{2}=0.659\right)$. As above, that GO and NOGO responses evolved in opposing directions is revealed in a highly significant (training stage*stimulus category) interaction term $(F(2,32)$ $=7.316, p=.002, \eta_{\mathrm{p}}^{2}=0.314$ ).

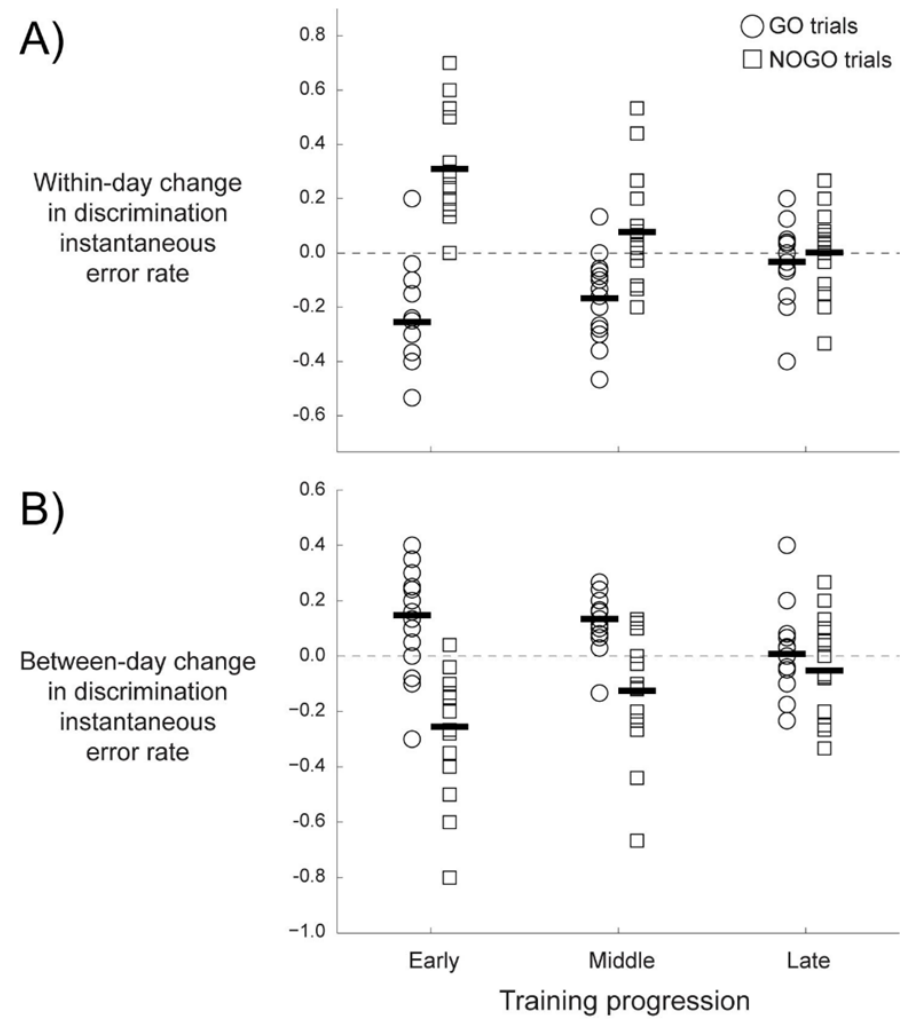

Figure 6. A) Changes in instantaneous error rates during the course of daily training differed over time and by stimulus identity. Each of the 17 trained subjects is represented by one symbol within each column; column means are indicated by thick bars. During the course of discrimination training, instantaneous response error rates during GO trials ( $\mathrm{o}$ symbols) improved (decreased) during daily training sessions, while instantaneous response error rates during NOGO trials ( $\square$ symbols) worsened (increased) during daily training. B) Instantaneous error rates changed overnight from the end of one training session to the start of the next training session in a manner that opposed, and at least partially offset, the pattern of daily changes. 


\section{Discussion}

We have demonstrated that zebra finches (males and females) experience gradual, overall improvements in GO-NOGO auditory discrimination performance across training sessions, a result which supports prior studies employing these or similar techniques (Appeltants, Gentner, Hulse, Balthazart, \& Ball, 2005; Gentner, Hulse, Bentley, \& Ball, 2000; Gess et al., 2011; Kriengwatana et al., 2016; Okanoya, Ikebuchi, Uno, \& Watanabe, 2001). We also have shown that these gradual daily improvements in overall task performance represent combinations of daily improvements in GO and NOGO discrimination that are quite distinct in their within- and across-day trajectories, as well as in their between-training session maintenance, a result which provides additional support and explanation for earlier demonstration of differential development of GO and NOGO learned responsiveness (Gess et al., 2011). Our findings suggest that the two components of this often-utilized discrimination task may be best described separately, rather than combined within a single outcomes metric as 'overall performance.' We present a descriptive model which depicts these different forms of learning as emerging from a combination of stimulus generalizability and subject modal response, and we propose that these differential learning trajectories are subserved by different neural learning systems, in concert with their different behavioral requirements.

The ability of zebra finches and other passerines to learn and perform an auditory discrimination task is at least partly a function of their having evolved a complicated neural network for the learning and use of vocal signals (Cynx \& Nottebohm, 1992; Doupe \& Konishi, 1991; Margoliash, 1997; Scharff et al., 1998; Theunissen, Amin, Shaevitz, Woolley, Fremouw, \& Hauber, 2004; Vates, Broome, Mello, \& Nottebohm, 1996). Unlike many other types of birds, passerines (as well as psittacines and trochiliformes) use auditory feedback from self-produced vocalizations to shape the form of their adult vocalizations, in a manner that parallels much of the phenomenology of human vocal learning (Bolhuis, Okanoya, \& Scharff, 2010; Doupe \& Kuhl, 1999; Jarvis, 2004; Marler, 1970). As such, the avian song neural system has been extensively studied, with distinct components of the auditory neural system populated by neurons that exhibit highly refined and selective responses to specific categories of auditory stimuli (Doupe \& Konishi, 1991; Konishi, 1985; Margoliash, 1983, 1986; McCasland \& Konishi, 1981; Theunissen et al., 2004; Williams \& Nottebohm, 1985). These studies offer evidence of a neural substrate by which auditory discrimination of vocal signals can occur. Although many of the neural underpinnings of avian vocal communication (such as song learning) are developmentally restricted in zebra finches, the ability to learn new auditory discriminations is not. Likewise, while only males of this species learn and use adult song, males and females should be expected to exhibit a high degree of auditory discrimination capability, as both sexes are expected to use male song in the context of individual assessments and interactions (Berglund, Bisazza, \& Pilastro, 1996; Catchpole, 1980; Kroodsma \& Byers, 1991; Nowicki $\&$ Searcy, 2005; Searcy, 1992). That females exhibit faster overall learning than do males may reflect the critical nature of vocal assessment in their mate selection and reproductive outcomes (Byers, Hebets, \& Podos, 2010; Byers \& Kroodsma, 2009; Catchpole, 1987; Kroodsma \& Byers, 1991; Nowicki \& Searcy, 2005; Searcy, 1992). That discrimination capability is adjustable through experience as well as seasonally plastic demonstrates that it is served by modifiable neural circuitry; our demonstration of differential evolution of learned GO and NOGO performance suggests that multiple of these plastic neural systems are engaged during discrimination learning.

Although the neural bases which underlie the different behavioral nature of GO and NOGO discrimination responses have received some attention (examples in Blake et al., 2002; Kalaska \& Crammond, 1995; Nieuwenhuis et al., 2003), the form and maintenance of their individual learning is rarely considered. Gess et al. (2011) suggested that individual response propensities could shape individual learning achievements, and they demonstrated select examples of the trajectories by which GO and NOGO task performance is achieved. Here we have a more-systematic result, in that all subjects exhibited differential GO-NOGO learning trajectories, of consistent forms, such that collective discrimination learning could be described by the separate achievements within GO and NOGO trials. Although subjects exhibited individual variation in the trajectories over which their task performances 
evolved, all subjects exhibited the same general pattern of differential GO and NOGO learning (Figure 4). As in any operant training, of course, the results here are contingency-dependent: three subjects that were trained on reverse contingencies (no reward for correct responses; Table 2) did not achieve daily progress in their task performance. However, even under these reverse contingencies, these three subjects exhibited very different GO and NOGO response performance trajectories (Figure 7), and all three subjects exhibited the same type of differential responsiveness (e.g., near-complete lack of performance during GO trials, nearly-perfect performance during NOGO trials).

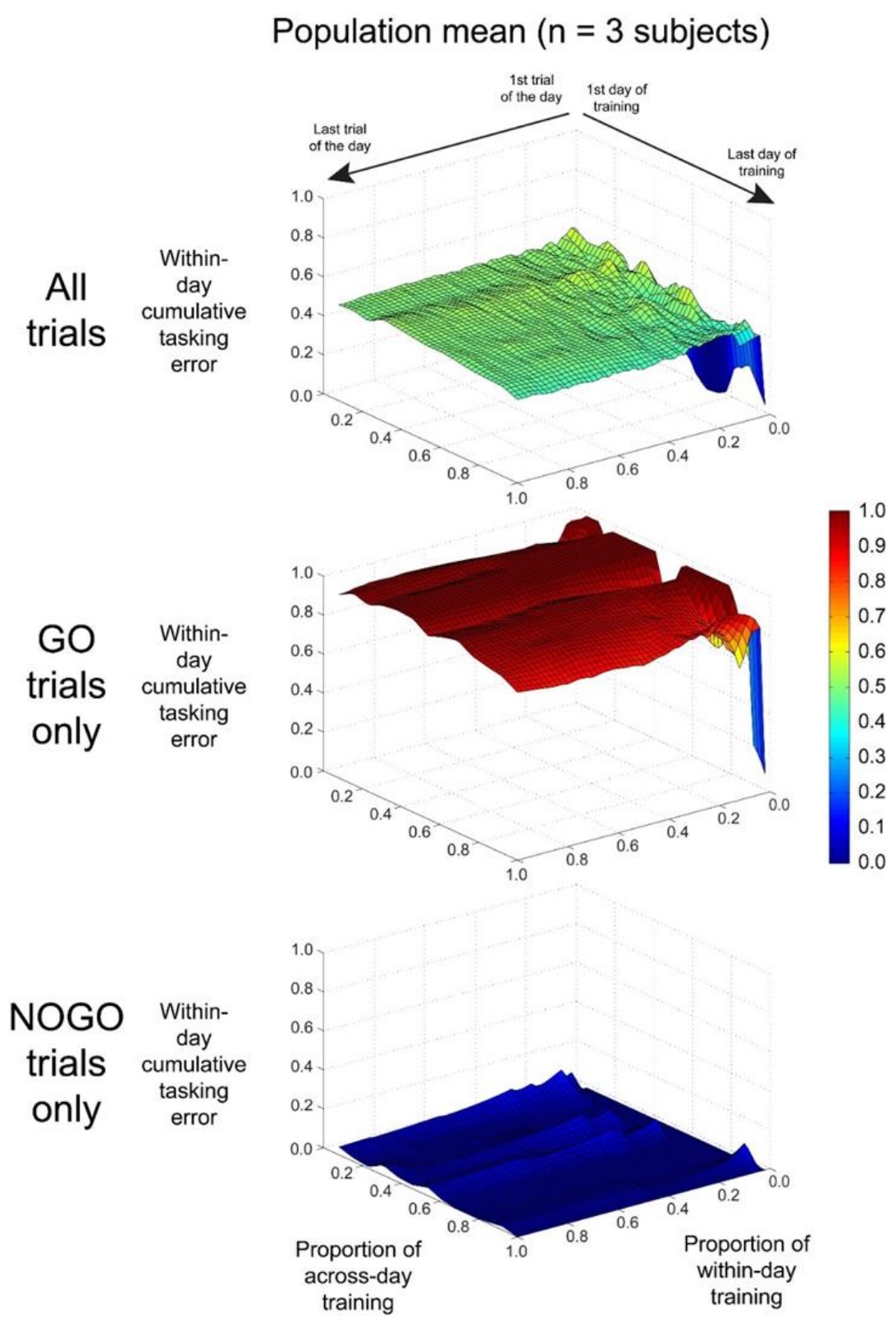

Figure 7. In order to confirm the ability of reward schedules to shape learning and evaluate the generality of differential GO and NOGO trial learning, three male subjects were trained with response contingencies that were the opposite of those normally used ('reverse contingencies'; Table 2). Under these contingencies, subject responses to sound stimuli quickly abated, and remained suppressed. This response pattern resulted in a lack of progress in discrimination learning (overall discrimination error remained ca. 50\%; figure top), with GO trial error rates fixed near 100\% (figure middle) and NOGO response error rates fixed near $0 \%$ (figure bottom). 
At some level, selection and motivation of any one behavior from a range of possible behaviors is a type of GO-NOGO response, in that the selected behavior must be motivated and executed while the non-selected behavior(s) must be suppressed (or, at minimum, not motivated). Mammalian studies have provided evidence of differential neural engagement during GO-NOGO tasking. For example, Kalaska and Crammond (1995) demonstrated differential pre-motor (but not parietal area 5) cortical activity during non-human primate GO-NOGO task performance, while Nieuwenhuis et al. (2003) reported strong event-related potential (ERP) signals from human anterior cingulate as a function of NOGO response withholding. These data demonstrate that GO-NOGO task performance utilizes neural circuits for both motor pattern selection/execution as well as for response evaluation/suppression. Models of basal ganglia function suggest that one of its primary functions toward voluntary behaviors is the selection of behavior among alternatives (Mink, 1996; Seger, 2008; Stephenson-Jones, Samuelsson, Ericsson, Robertson, \& Grillner, 2011), which would strongly implicate basal ganglia contributions toward GO-NOGO task performance. Similarly, the rewards associated with correct GO responses could be expected to trigger neural affective and pre-motor planning pathways. Given that individuals in our version of this task controlled their own work rate, they may be expected to only perform when reward is desired, suggesting that motivation is a primary contributor toward task initiation. It may be that high levels of motivation (which are necessary for trial initiation in this version of the task) also contribute to high levels of GO trial response error, especially during the middle of training when neophobia toward the training environment has decreased, and when the number of response successes are low and thus rewards (both food and neural) are relatively more beneficial. Gess et al. (2011) also reported high levels of GO trial trigger activation in their experiments (with select examples presented in their Figure 4), which suggests that discrimination task performance that is motivation based could be biased toward 'GO-type' responses.

Our pattern of results suggests that multiple drivers of behavior contribute to the evolving nature of differential GO-NOGO learned responsiveness. We have synthesized (Table 3) our results to demonstrate how two of the fundamental criteria which underlie this type of discrimination (motivational responsiveness and true discrimination capability, neither of which are directly measured here) may combine to produce the behavioral result that is directly evaluated here (observed discrimination capability). Together, these two factors interact in a manner which describes well the patterns of response performance observed here. Early in training, subjects initiated discrimination trials at high rates, but exhibited little ability to perform the discrimination and typically failed to respond to triggered stimuli. At this point in training, the modal response to stimuli was 'inaction', producing high GO trial error rates and low NOGO error rates. This is not unexpected, as a lack of familiarity with the task and the stimuli make correct discrimination (and behavioral reporting of discrimination capability) at the start of training highly unlikely.

Table 3

Evolving Stimulus Generalizability and Modal Response Together Predict Differential GO and NOGO Training Progression

\begin{tabular}{ccccc}
\hline Training phase & \multicolumn{3}{c}{ Across Day Training Progression } \\
& & Early & Middle & Late \\
\hline Overall discrimination capability & & High & Low & High \\
Generalization of responses & High & High & Low \\
Modal response to stimuli & Inaction & Action & Mixed \\
GO trial error & High & Moderate & Low \\
NOGO trial error & Low & Moderate & Low \\
Overall trial error & GO trial error & Moderate & Moderate & Low \\
Magnitude of daily change & NOGO trial error & +-+ & - & + \\
Magnitude of nightly change & GO trial error & ++ & ++ & 0 \\
& NOGO trial error & -- & + & 0 \\
\hline
\end{tabular}


After several training sessions, subjects appeared to better understand the mechanics of the task. During the middle of training, our results suggest that the stimuli are still generalized (e.g., not well discriminated), but now the modal response to stimuli is 'action' (activation of the response trigger). This produces moderate levels of both GO and NOGO trial error, as observed. Late in training, once stimulus discrimination is achieved, responses are no longer generalized. Rather, response form is then tailored to stimulus type, producing relatively low rates of both GO and NOGO error. Similarly, the initial response bias exhibited by subjects in the study by Gess et al. (2011) was also seen to fade through training, as subject responses came under stimulus control. Our descriptive model accurately captures the pattern of differential GO-NOGO response learning that we have observed, and it matches the gradual improvement in overall task performance that we and others have reported. Importantly, our descriptive model suggests that overall task performance improves more in the latter than in the early stages of training (in agreement with the results we have observed; Figure 5, left), and suggests that subjects' ability to discriminate stimuli is a primary, but not the only, determinant of task performance.

Our data also reveal that the magnitude, and direction, of daily improvements in task performance are partially offset by between-session (e.g., overnight) losses in performance. This finding is consistent with that reported in phenomenological studies of bird song motor learning (Derégnaucourt, Mitra, Fehér, Pytte, \& Tchernichovski, 2005), bumblebee foraging (Keasar, Motro, Shur, \& Shmida, 1996), and for some types of human memory recall (Wilhelm, Diekelmann, \& Born, 2008). These findings also are matched by studies of neuronal consolidation, which, for some types of skills, generally report an imperfect ability to translate short-term gains in behavioral performance into long-term, more-permanent modifications of neural circuitry (reviews in Schönauer, Grätsch, \& Gais, 2015; Straube, 2012; Walker \& Stickgold, 2004). Investigation of the neural mechanisms by which one task response (NOGO trials) improves overnight while another (GO response) worsens will be required to determine the degree to which different neural circuits bear primary responsibility for the organization, improvement, and maintenance of these opposing responses. Human imaging studies suggest that GO-NOGO task performance in humans engages a wide array of neural structures, including attentional, memory, evaluative, and pre-motor areas (Criaud \& Boulinguez, 2013). Our current data suggest that animal discrimination performance may be similarly complex, such that neural investigations in animal models of the differential mechanisms involved in GO and NOGO responsiveness would be fruitful. For example, reward schedules are well understood to shape both neural and behavioral responses to stimuli in general (example in Fiorillo, Tobler, \& Schultz, 2003). Our results suggest that they can be expected to do so differentially within this type of discrimination task (with an expectation of differing impacts on GO and NOGO responsiveness) through modulation of neural reward circuitry. Another opportunity for further study involves evaluating the long-term maintenance of responses, without which improvement gains are lost. Our results demonstrate that learned GO and NOGO responses are maintained very differently between sessions (overnight), suggesting that they are subject to differential neural consolidation. Impaired memory consolidation is known to reduce discrimination and motor task performance gains through disruption of normal sleep (reviewed in Stickgold, 2005) or through direct molecular blockade of protein synthesis (reviewed by Pearce, Cai, Roberts, \& Glanzman, 2017). We also expect that disruption of the consolidation of daily changes in task performance would impact GO and NOGO performance in unequal ways. Many experimental paradigms have been developed to explore behavioral and molecular influences on (overall) discrimination task performance; application of these established experimental schemes with an eye toward differential GO and NOGO learned responsiveness would clarify the relative importance of neural reward, behavioral motivation, and molecular memory in the independent emergence of GO and NOGO responses during the acquisition of overall discrimination capability. 


\section{Acknowledgements}

This work was supported by the IUP School of Graduate Studies and Research, and by research awards to PMN from the Pennsylvania Lions Hearing Research Foundation. We thank Matt Reitzel for technical assistance, and William Farrell and Cora Lou Sherburne for discussion and advice.

\section{References}

Ahmadi A., Pearlson, G. D., Meda, S. A., Dager, A., Potenza, M. N., Rosen, R., ...Stevens, M. C. (2013). Influence of alcohol use on neural response to go/no-go task in college drinkers. Neuropsychopharmacology, 38, 2197-2208. http://dx.doi.org/10.1038/npp.2013.119

Appeltants, D., Gentner, T. Q., Hulse, S. H., Balthazart, J., \& Ball, G. F. (2005). The effect of auditory distractors on song discrimination in male canaries (Serinus canaria). Behavioural Processes, 69, 331-341. https://doi.org/10.1016/j.beproc.2005.01.010

Berglund, A., Bisazza, A., \& Pilastro, A. (1996). Armaments and ornaments: An evolutionary explanation of traits of dual utility. Biological Journal of the Linnean Society, 58, 385-399. http://dx.doi.org/10.1111/j.10958312.1996.tb01442.x

Blake, D. T., Strata, F., Churchland, A. K., \& Merzenich, M. M. (2002). Neural correlates of instrumental learning in primary auditory cortex. Proceedings of the National Academy of Sciences, 99, 10114-10119. http://dx.doi.org/10.1073/pnas.092278099

Bolhuis, J. J., Okanoya, K., \& Scharff, C. (2010). Twitter evolution: Converging mechanisms in birdsong and human speech. Nature Reviews Neuroscience, 11, 747-759. http://dx.doi.org/10.1038/nrn2931

Byers, B. E., \& Kroodsma, D. E. (2009). Female mate choice and songbird song repertoires. Animal Behaviour, 77, 13-22. https://doi.org/10.1016/j.anbehav.2008.10.003

Byers, J., Hebets, E., \& Podos, J. (2010). Female mate choice based upon male motor performance. Animal Behaviour, 79, 771-778. https://doi.org/10.1016/j.anbehav.2010.01.009

Catchpole, C. K. (1980). Sexual selection and the evolution of complex songs among European warblers of the genus Acrocephalus. Behaviour, 74, 149-165. https://doi.org/10.1163/156853980X00366

Catchpole, C. K. (1987). Bird song, sexual selection and female choice. Trends in Ecology \& Evolution, $2,94-97$. https://doi.org/10.1016/0169-5347(87)90165-0

Criaud, M., \& Boulinguez, P. (2013). Have we been asking the right questions when assessing response inhibition in go/no-go tasks with fMRI? A meta-analysis and critical review. Neuroscience \& Biobehavioral Reviews, 37, 11-23. http://dx.doi.org/10.1016/j.neubiorev.2012.11.003

Cynx, J. (1993). Conspecific song perception in zebra finches (Taeniopygia guttata). Journal of Comparative Psychology, 107, 395-402. http://dx.doi.org/10.1037/0735-7036.107.4.395

Cynx, J., \& Nottebohm, F. (1992). Role of gender, season, and familiarity in discrimination of conspecific song by zebra finches (Taeniopygia guttata). Proceedings of the National Academy of Sciences, 89, 1368-1371. http://dx.doi.org/10.1073/pnas.89.4.1368

Cynx, J., Williams, H., \& Nottebohm, F. (1992). Hemispheric differences in avian song discrimination. Proceedings of the National Academy of Sciences, 89, 1372-1375. http://dx.doi.org/10.1073/pnas.89.4.1372

de Haan, R., Lim, J., van der Burg, S. A., Pieneman, A. W., Nigade, V., Mansvelder, H. D., \& de Kock, C. P. J. (2018). Neural representation of motor output, context and behavioral adaptation in rat medial prefrontal cortex during learned behavior. Frontiers in Neural Circuits 12, 75. http://dx.doi.org/10.3389/fncir.2018.00075

Derégnaucourt, S., Mitra, P. P., Fehér, O., Pytte, C., \& Tchernichovski, O. (2005). How sleep affects the developmental learning of bird song. Nature, 433, 710-716. http://dx.doi.org/10.1038/nature03275

Doupe, A. J., \& Konishi, M. (1991). Song-selective auditory circuits in the vocal control system of the zebra finch. Proceedings of the National Academy of Sciences, 88, 11339-11343. http://dx.doi.org/10.1073/pnas.88.24.11339

Doupe, A. J., \& Kuhl, P. K. (1999). Birdsong and human speech: Common themes and mechanisms. Annual Review of Neuroscience, 22, 567-631. http://dx.doi.org/10.1146/annurev.neuro.22.1.567

Fiorillo, C. D., Tobler, P. N., \& Schultz, W. (2003). Discrete coding of reward probability and uncertainty by dopamine neurons. Science, 299, 1898-1902. http://dx.doi.org/10.1126/science.1077349 
Gentner, T. Q., Hulse, S. H., Bentley, G. E., \& Ball, G. F. (2000). Individual vocal recognition and the effect of partial lesions to $\mathrm{HVc}$ on discrimination, learning, and categorization of conspecific song in adult songbirds. Journal of Neurobiology, 42, 117-133. http://dx.doi.org/10.1002/(SICI)1097-4695(200001)42:1\%3C117::AID-NEU11\%3E3.0.CO;2-M

Gess, A., Schneider, D. M., Vyas, A., \& Woolley, S. M. N. (2011). Automated auditory recognition training and testing. Animal Behaviour, 82, 285-293. http://dx.doi.org/10.1016/j.anbehav.2011.05.003

Jarvis, E. D. (2004). Learned birdsong and the neurobiology of human language. Annals of the New York Academy of Sciences, 1016, 749-777. http://dx.doi.org/10.1196/annals.1298.038

Kalaska, J. F., \& Crammond, D. J. (1995). Deciding not to go: Neuronal correlates of response selection in a go/nogo task in primate premotor and parietal cortex. Cerebral Cortex, 5, 410-428. http://dx.doi.org/10.1093/cercor/5.5.410

Keasar, T., Motro, U. Z. I., Shur, Y., \& Shmida, A. V. I. (1996). Overnight memory retention of foraging skills by bumblebees is imperfect. Animal Behaviour, 52, 95-104. https://doi.org/10.1006/anbe.1996.0155

Konishi, M. (1985). Birdsong: From behavior to neuron. Annual Review of Neuroscience, 8, 125-170. http://dx.doi.org/10.1146/annurev.ne.08.030185.001013

Kraemer, S., \& Apfelbach, R. (2004). Olfactory sensitivity, learning and cognition in young adult and aged male Wistar rats. Physiology \& Behavior, 81, 435-442. http://dx.doi.org/10.1016/j.physbeh.2004.01.012

Kriengwatana, B., Spierings, M. J., \& ten Cate, C. (2016). Auditory discrimination learning in zebra finches: Effects of sex, early life conditions and stimulus characteristics. Animal Behaviour, 116, 99-112. https://doi.org/10.1016/j.anbehav.2016.03.028

Kroodsma, D. E., \& Byers, B. E. (1991). The function(s) of bird song. American Zoologist, 31, 318-328. http://dx.doi.org/10.1093/icb/31.2.318

Margoliash, D. (1983). Acoustic parameters underlying the responses of song-specific neurons in the white-crowned sparrow. The Journal of Neuroscience, 3, 1039-1057. http://dx.doi.org/10.1523/JNEUROSCI.03-0501039.1983

Margoliash, D. (1986). Preference for autogenous song by auditory neurons in a song system nucleus of the whitecrowned sparrow. The Journal of Neuroscience, 6, 1643-1661. http://dx.doi.org/10.1523/JNEUROSCI.0606-01643.1986

Margoliash, D. (1997). Functional organization of forebrain pathways for song production and perception. Journal of Neurobiology, 33, 671-693. http://dx.doi.org/10.1002/(sici)1097-4695(19971105)33:5<671::aid-neu12>3.0.co;2-c

Marler, P. (1970). Birdsong and speech development: Could there be parallels? American Scientist, 58, 669-673. http://www.jstor.org/stable/27829317

McCasland, J. S., \& Konishi, M. (1981). Interaction between auditory and motor activities in an avian song control nucleus. Proceedings of the National Academy of Sciences, 78, 7815-7819. http://dx.doi.org/10.1073/pnas.78.12.7815

Mink, J. W. (1996). The basal ganglia: Focused selection and inhibition of competing motor programs. Progress in Neurobiology, 50, 381-425. https://doi.org/10.1016/s0301-0082(96)00042-1

Nieuwenhuis, S., Yeung, N., van den Wildenberg, W., \& Ridderinkhof, K. R. (2003). Electrophysiological correlates of anterior cingulate function in a go/no-go task: Effects of response conflict and trial type frequency. Cognitive, Affective, \& Behavioral Neuroscience, 3, 17-26. http://dx.doi.org/10.3758/CABN.3.1.17

Nowicki, S., \& Searcy, W. A. (2005). Song and mate choice in birds: How the development of behavior helps us understand function. The Auk, 122, 1-14. http://dx.doi.org/10.1642/00048038(2005)122\%5B0001:SAMCIB\%5D2.0.CO;2

Okanoya, K., \& Dooling, R. J. (1991a). Detection of species-specific calls in noise by zebra finches Poephila guttata and budgerigars Melopsittacus undulatus: Time of frequency domain? Bioacoustics, 3, 163-172. http://dx.doi.org/10.1080/09524622.1991.9753177

Okanoya, K., \& Dooling, R. J. (1991b). Perception of distance calls by budgerigars (Melopsittacus undulatus) and zebra finches (Poephila guttata): Assessing species-specific advantages. Journal of Comparative Psychology, 105, 60-72. http://dx.doi.org/10.1037/0735-7036.105.1.60

Okanoya, K., Ikebuchi, M., Uno, H., \& Watanabe, S. (2001). Left-side dominance for song discrimination in Bengalese finches (Lonchura striata var. domestica). Animal Cognition, 4, 241-245. http://dx.doi.org/10.1007/s10071-001-0120-9 
Pearce, K., Cai, D., Roberts, A. C., \& Glanzman, D. L. (2017). Role of protein synthesis and DNA methylation in the consolidation and maintenance of long-term memory in Aplysia. eLife, 6, e18299. http://dx.doi.org/10.7554/eLife.18299

Remington, E. D., Osmanski, M. S., \& Wang, X. Q. (2012). An operant conditioning method for studying auditory behaviors in marmoset monkeys. PLOS One, 7, e47895. https://doi.org/10.1371/journal.pone.0047895

Riebel, K., \& Slater, P. J. B. (1998). Testing female chaffinch song preferences by operant conditioning. Animal Behaviour, 56, 1443-1453. https://doi.org/10.1006/anbe.1998.0933

Scharff, C., Nottebohm, F., \& Cynx, J. (1998). Conspecific and heterospecific song discrimination in male zebra finches with lesions in the anterior forebrain pathway. Journal of Neurobiology, 36, 81-90. http://dx.doi.org/10.1002/(sici)1097-4695(199807)36:1<81::aid-neu7>3.0.co;2-6

Schellinck, H. M., Brown, R. E., \& Slotnick, B. M. (1991). Training rats to discriminate between the odors of individual conspecifics. Animal Learning \& Behavior, 19, 223-233. http://dx.doi.org/10.3758/Bf03197880

Schiff, H., Bouhuis, A. L., Yu, K., Penzo, M. A., Li, H., He, M., \& Li, B. (2018). An insula-central amygdala circuit for guiding tastant-reinforced choice behavior. The Journal of Neuroscience, 38, 1418-1429. http://dx.doi.org/10.1523/JNEUROSCI.1773-17.2017

Schönauer, M., Grätsch, M., \& Gais, S. (2015). Evidence for two distinct sleep-related long-term memory consolidation processes. Cortex, 63, 68-78. https://doi.org/10.1016/j.cortex.2014.08.005

Searcy, W. A. (1992). Song repertoire and mate choice in birds. American Zoologist, 32, 71-80. http://dx.doi.org/10.1093/icb/32.1.71

Seger, C. A. (2008). How do the basal ganglia contribute to categorization? Their roles in generalization, response selection, and learning via feedback. Neuroscience \& Biobehavioral Reviews, 32, 265-278. https://doi.org/10.1016/j.neubiorev.2007.07.010

Simson, R., Vaughan, H. G., Jr., \& Ritter, W. (1977). The scalp topography of potentials in auditory and visual go/nogo tasks. Electroencephalography and Clinical Neurophysiology, 43, 864-875. http://dx.doi.org/10.1016/0013-4694(77)90009-8

Skinner, B. F. (1963). Operant behavior. American Psychologist, 18, 503-515. http://dx.doi.org/10.1037/h0045185

Sossinka, R., \& Boehner, J. (1980). Song types in the zebra finch Poephila guttata castanotis. Zeitschrift für Tierpsychologie 53, 123-132. https://doi.org/10.1111/j.1439-0310.1980.tb01044.x

Stephenson-Jones, M., Samuelsson, E., Ericsson, J., Robertson, B., \& Grillner, S. (2011). Evolutionary conservation of the basal ganglia as a common vertebrate mechanism for action selection. Current Biology, 21, 10811091. https://doi.org/10.1016/j.cub.2011.05.001

Stickgold, R. (2005). Sleep-dependent memory consolidation. Nature, 437, 1272-1278. http://dx.doi.org/10.1038/nature04286

Straube, B. (2012). An overview of the neuro-cognitive processes involved in the encoding, consolidation, and retrieval of true and false memories. Behavioral and Brain Functions, 8, 35. http://dx.doi.org/10.1186/1744-9081-8-35

Theunissen, F. E., Amin, N., Shaevitz, S. S., Woolley, S. M. N., Fremouw, T., \& Hauber, M. E. (2004). Song selectivity in the song system and in the auditory forebrain. Annals of the New York Academy of Sciences, 1016, 222-245. http://dx.doi.org/10.1196/annals.1298.023

Vates, G. E., Broome, B. M., Mello, C. V., \& Nottebohm, F. (1996). Auditory pathways of caudal telencephalon and their relation to the song system of adult male zebra finches (Taenopygia guttata). Journal of Comparative Neurology, 366, 613-642. http://dx.doi.org/10.1002/(sici)1096-9861(19960318)366:4<613::aidcne5>3.0.co;2-7

Verzijden, M. N., Etman, E., van Heijningen, C., van der Linden, M., \& ten Cate, C. (2007). Song discrimination learning in zebra finches induces highly divergent responses to novel songs. Proceedings of the Royal Society B: Biological Sciences, 274, 295-301. http://dx.doi.org/10.1098/rspb.2006.3728

Walker, M. P., \& Stickgold, R. (2004). Sleep-dependent learning and memory consolidation. Neuron, 44, 121-133. https://doi.org/10.1016/j.neuron.2004.08.031

Wilhelm, I., Diekelmann, S., \& Born, J. (2008). Sleep in children improves memory performance on declarative but not procedural tasks. Learning \& Memory, 15, 373-377. http://dx.doi.org/10.1101/lm.803708

Williams, H., \& Nottebohm, F. (1985). Auditory responses in avian vocal motor neurons: A motor theory for song perception in birds. Science, 229, 279-282. http://dx.doi.org/10.1126/science.4012321 